\title{
"Is it a Curse to Suffer from Cancer in Sub-Saharan Africa?": The Situation of Radiation Therapy for Management of Head and Neck Cancers
}

\author{
Rasheed Ofosu-Poku*, Alberta Delali Dzaka and Gladys Anyane \\ Directorate of Family Medicine, Komfo Anokye Teaching Hospital, Kumasi, Ghana \\ *Corresponding Author: Rasheed Ofosu-Poku, Directorate of Family Medicine, \\ Komfo Anokye Teaching Hospital, Kumasi, Ghana.
}

Received: September 21, 2021

Published: September 24, 2021

(C) All rights are reserved by Rasheed

Ofosu-Poku., et al.

\section{Introduction}

Head and neck cancers are a heterogeneous group of cancers affecting the oral cavity, nasal cavity, pharynx, larynx, paranasal sinuses and salivary glands $[1,2]$. The incidence of head and neck cancers are increasing globally, especially in low and middle income countries $[3,4]$. Mortality and morbidity from head and neck cancers are also increasing in low and middle income countries on account of their poor biomedical resource availability and accessibility, and health-seeking behaviour [5-8].

\section{Accessibility to radiotherapy in Sub-Saharan Africa}

In the management of head and neck cancers, radiotherapy plays a significant role irrespective of the stage of presentation, whether the intent of care is cure or palliation [1,9]. It is recommended that for developing countries, every population of 1 million patients require 1 - 3 radiotherapy installations to meet their health care needs [10].

However, $50 \%$ of patients in lower-middle income countries and $90 \%$ in low income countries who need radiotherapy services never get access to it [11]. A review of the population of sub-Sahara African states reveals the grossly inadequate radiotherapy resources available to its people. For instance, Nigeria has eight (8) radiotherapy installations for a population of 186 million [12], Ghana has three (3) for 31 million people $[10,13]$ and Burkina Faso one (1) for 20 million people [14,15]. Thus, in cancer treatment centres in this region, clinicians may be forced to prioritize re- sources for patients who are more likely to get the greatest benefit from radiotherapy whilst depriving the others.

Another challenge affecting most sub-Saharan African states is the frequent breakdown in radiotherapy installations [16]. On account of the limited numbers, there is increased pressure on those available leading to frequent breakdown. Secondly, the needed human resource and equipment parts to fix identified faults are unavailable and mostly needs to be imported.

These challenges cause patients with head and neck cancers on radiotherapy to have interrupted treatment which may lead to poor treatment outcomes. In some situations, the radiotherapy equipment gets repaired after the condition of patients may have deteriorated with reduced performance status and patients being ineligible to receive radiotherapy. The result of this situation is huge financial losses as patients sometimes make full payment before starting the radiotherapy but end up not receiving the planned quantity, they played for.

A reflection on the condition of these patients who may have benefited from radiotherapy but continue to suffer unduly on account of the frequent breakdown of radiotherapy equipment and their inadequate numbers in sub-Saharan Africa elicits various questions such as: 'Is it a curse to suffer from cancer in sub-Saharan Africa?', 'If this patient were in a developed country, will his/ her care and treatment not be better because of the accessibility 
to radiotherapy services?', 'Why is the country not having enough radiotherapy services to meet the demands of the population?'

\section{Recommendations}

Governments and policy makers in sub-Saharan African states must prioritize the health and wellbeing of their people by ensuring that needed resources and equipment such as radiotherapy machines of high quality are made available in sufficient numbers at strategic points in their respective countries, to improve accessibility and ensure equitable distribution of essential radiotherapy services to all their citizens.

Biomedical engineers should be trained in sufficient numbers. Agreements with companies that make these radiotherapy machines should be made prior to or when they are being acquired, to have biomedical engineers from the country undergo further training about those equipment and how to deal with any faults they may develop. Replacement parts of the radiotherapy machines should be stocked in the medical stores of hospitals in which they are found so that they can be appropriately repaired when they become faulty.

\section{Bibliography}

1. DG Pfister., et al. "NCCN Clinical Practice Guidelines in Oncology: Head and Neck Cancers, Version 2.2020". Journal of National Comprehensive Cancer Network 18.7 (2020).

2. O Owusu-Afriyie., et al. "Survival rates of head and neck cancers in Ghana: a retrospective study of the Komfo Anokye Teaching Hospital”. BMC Research Notes 13 (2020): 392.

3. AE Abdulai., et al. "Primary malignant head and neck tumours in Ghana: A survey of histopathological charts over two decades". International Journal of Medicine and Biomedical Research 2.1 (2013).

4. B Gupta., et al. "Global epidemiology of head and neck cancers: A continuing challenge". Oncology 91 (2016): 13-23.

5. World Health Organization, "Low quality healthcare is increasing the burden of illness and health costs globally". World Health Organization (2018).
6. SE Hills and ME Prince. "Quality of life of head and neck cancer patients in Ghana". Otolaryngology-Head and Neck Surgery 147 (2012): 2S.

7. S Kamaraju., et al. "Cancer prevention in low-resource countries: An overview of the opportunity". American Society of Clinical Oncology Educational Book 30 (2020): 1-12.

8. VZ Kuuire., et al. "Health-seeking behaviour during times of illness: A study among adults in a resource poor setting in Ghana”. Journal of Public Health 38.4 (2016): E545-e553.

9. AS Grewal., et al. "Palliative radiation therapy for head and neck cancers". International Journal of Radiation Oncology, Biology, Physics 105.2 (2019): 254-266.

10. V Vanderpuye and NA Aryeetey. "Cancer radiotherapy in Ghana". Cancer Control (2016).

11. EH Zubizarreta., et al. "Need for radiotherapy in low and middle income countries: The silent crisis continues". Clinical Oncology 27 (2015): 107-114.

12. CS Aruah. "The current state of radiotherapy practice in Nigeria: What the future hold". European Organisation for Nuclear Research (CERN) (2017).

13. The World Bank, "Population, total - Ghana". The World Bank (2021).

14. The World Bank, "Population, total - Burkina Faso". The World Bank (2021).

15. Dear Mamma "Burkina Faso gets new treatment centre". Dearmama (2021).

16. Komfo Anokye Teaching Hospital (KATH), "KATH Annual Report 2017". KATH (2018).

\section{Volume 3 Issue 10 October 2021 (C) All rights are reserved by Rasheed Ofosu-Poku., et al.}

